\title{
openheart Importance of maintaining a low omega-6/omega-3 ratio for reducing platelet aggregation, coagulation and thrombosis
}

To cite: DiNicolantonio JJ, OKeefe J. Importance of maintaining a low omega-6/ omega-3 ratio for reducing platelet aggregation, coagulation and thrombosis. Open Heart 2019;6:e001011. doi:10.1136/ openhrt-2019-001011

Accepted 14 April 2019

Check for updates

C Author(s) (or their employer(s)) 2019. Re-use permitted under CC BY-NC. No commercial re-use. See rights and permissions. Published by BMJ.

${ }^{1}$ Department of Preventive Cardiology, Mid America Heart Institute, Kansas City, Missouri, USA

${ }^{2}$ Saint Luke's Mid America Heart Institute, University of MissouriKansas City, Kansas City,

Missouri, USA

Correspondence to Dr James J DiNicolantonio; jjdinicol@gmail.com
The idea that long-chain marine omega-3s can prevent cardiovascular disease is decades old. This idea began with the Greenland Inuit who were noted to have a lower risk of cardiovascular disease. ${ }^{1}$ Indeed, the Inuit, with their high intake of long-chain omega-3s, have been noted to have a lower platelet count, reduced platelet reactivity, prolonged bleeding times and a lower ratio of proaggregatory thromboxanes versus antiaggregatory prostacyclins. ${ }^{2}$ And it has been known for a long time that marine omega-3s (from salmon, mackerel, fish oil or cod liver oil) inhibit platelet aggregation.

\section{OMEGA-3S REDUCE PLATELET AGGREGATION, COAGULATION AND THROMBOSIS}

Clinical studies in humans clearly show that marine omega-3s provide antiplatelet effects. Indeed, a meta-analysis of 15 randomised controlled trials (RCT) in humans has confirmed that omega-3 polyunsaturated fatty acids (PUFA) inhibit platelet aggregation. ${ }^{3}$ Marine omega-3 PUFAs also may help overcome aspirin resistance. ${ }^{4}$ In healthy borderline overweight men, $3 \mathrm{~g}$ of omega-3 PUFAs for 4 weeks lowered fibrinogen, thrombin and factor V levels; these benefits occurred mainly in those with high fibrinogen carrying alpha-chain fibrinogen polymorphism. ${ }^{5}$

Marine omega-3s also have the ability to reduce von Willebrand factor (vWF; a platelet activator factor), whole blood viscosity, and can improve red blood cell flexibility (deformability). ${ }^{6} 7$ In a 5 -week double-blind placebo-controlled study in 30 healthy subjects, $2.52 \mathrm{~g} /$ day of omega-3 PUFAs as compared with $1.26 \mathrm{~g} /$ day, significantly decreased plasma viscosity, red blood cell rigidity and systolic blood pressure. ${ }^{8}$ Thus, higher doses of marine omega-3 seem to be more effective antithrombotic benefits.
One study in healthy adults found that fish oil (providing $6 \mathrm{~g}$ of eicosapentaenoic acid (EPA)/day), but not vegetable oil, reduced platelet adhesiveness. ${ }^{9}$ In another study, supplementation with $3.6 \mathrm{~g}$ of omega-3 PUFA from fish oil reduced platelet aggregation, whereas $25 \mathrm{~g}$ of soy lecithin (providing $1.5 \mathrm{~g}$ omega-6, 0.5 g omega-3) increased platelet reactivity; no effect was found in the control group. ${ }^{10}$ The omega- $6 /$ omega- 3 ratio in platelets is also positively correlated with platelet adhesion at rest and after ADP and thrombin platelet stimulation. Another study found that plasminogen activator inhibitor-1 (PAI-1, an inhibitor of fibrinolysis) can be lowered in those consuming fish oil, suggesting a decreased risk of thrombosis. ${ }^{11}$

In general, approximately 2-4 $\mathrm{g}$ of EPA/ docosahexaenoic acid (DHA) per day is needed to provide the full antiatherosclerotic, anti-inflammatory and antiplatelet benefits. ${ }^{12}$ Even plant omega-3s seem to have some benefit in this regard, whereas omega- 6 may have a detrimental effect. Indeed, on a diet high in monounsaturated fatty acids (MUFA), as the omega-6 linoleic acid (LA)/ omega-3 alpha linolenic acid (ALA) ratio decreases, platelet aggregation decreases. ${ }^{13}$ In vitro platelet aggregation to both $\mathrm{ADP}$ and collagen is even increased after sunflower and rapeseed oil compared with a diet enriched in milk fat. ${ }^{14}$ This suggests that even compared with saturated fat, a diet high in omega-6 PUFA may actually increase platelet aggregation.

In 24 healthy young males, a Mediterranean diet has been found to reduce the thrombotic state (decreased plasma vWF, tissue factor pathway inhibitor and tissue PAI-1). ${ }^{15}$ Oleic acid may provide similar, but slightly less antiplatelet effects as long-chain marine omega-3s since the omega-9 fatty acid eicosatrienoic acid has also been found to reduce 
the production of thromboxane-B2 (TXB2), which is the inactivated metabolite of TXA2 (a platelet activator). ${ }^{16}$ Moreover, in another study, TXB2 production in platelets was reduced with olive oil supplementation but not with a corn oil-enriched diet. ${ }^{16}$ Animal studies confirm a reduction in TXB2 with the use of olive oil, an effect which is greater than that found with sunflower oil. ${ }^{17}$ Another study found a reduction in thromboxane production (urinary excretion of the TXB2 metabolite 11-dehydro-TXB2) with saturated fat and MUFA versus omega-6 PUFA. ${ }^{18}$ A Mediterranean diet high in MUFA reduces $\mathrm{VWF}$ (which is derived from the endothelium and is important in the coagulation process during a platelet thrombus) and PAI-1. ${ }^{1519}$

The type of long-chain marine omega-3 may also affect the antiplatelet effects of marine omega-3s. Indeed, platelet aggregation in response to collagen is reduced in just 6 days after pure EPA consumption but platelet response to ADP is not reduced until after at least 4 weeks of intake; however, the inhibition of platelet aggregation with DHA ( $6 \mathrm{~g} /$ day) to both stimuli occurs in just 6 days. ${ }^{20}$ Thus, both EPA and DHA inhibit platelet aggregation; however, DHA has a faster onset of action in regard to inhibiting ADP-induced platelet aggregation.

Both EPA and DHA get incorporated into platelet phospholipids at the expense of arachidonic acid (AA), which may help reduce platelet aggregation via a reduction in AA-derived platelet-aggregating/procoagulant metabolites. Additionally, EPA competes with AA for cyclo-oxygenase reducing its action on AA. Thus, EPA both directly and indirectly reduces the formation of the AA proaggregatory metabolite TXA2. ${ }^{20} \mathrm{EPA} / \mathrm{DHA}$ also gets incorporated into neutrophils and red blood cells at the expense of both LA and AA. The incorporation of omega-3s in red blood cells seems to decrease whole blood viscosity and increase red blood cell flexibility thus likely reducing the risk of thrombosis. ${ }^{67}$

Daily supplementation with $3 \mathrm{~g}$ of EPA/DHA for 12 weeks, and especially after 18 weeks, inhibits tissue factor activity in adherent monocytes (a catalyst in the coagulation cascade); this benefit also occurs after 24 weeks in those with hypertriglyceridaemia. ${ }^{21}$ Thus, the antithrombotic effects of omega-3s in clinical studies may need to be tested for a minimum of 18 weeks in healthy patients and even longer (for 24 weeks) in those with hypertriglyceridaemia.

One study in healthy young men found that both lean meat and fish have antithrombotic effects although some prothrombotic effects (such as an increase in PAI-1) were also noted with increased fish intake. ${ }^{22} \mathrm{~A}$ decrease in platelet aggregation but an increase in PAI-1 has sometimes been noted with fish oil supplementation. This increase in PAI-1 may actually occur to naturally counteract any excessive inhibition in the coagulation cascade, which is why supplementing with marine omega-3s is not associated with a significant increase in major or clinically significant bleeds. And considering that marine omega-3s consistently lower the risk of thrombotic events, ${ }^{12}{ }^{23}$ there does not appear to be increased coagulation but a decrease. Interestingly, supplementing the diet with olive oil or consuming fish on top of a MUFA-enriched Mediterranean diet has been found to decrease PAI-1. ${ }^{15} 24$

One double-blind placebo-controlled trial in 59 patients with hypertension with type 2 diabetes compared $4 \mathrm{~g}$ /day of EPA, $4 \mathrm{~g} /$ day of DHA or $4 \mathrm{~g} /$ day of olive oil ('placebo') for 6 weeks. ${ }^{25}$ DHA, but not EPA, significantly reduced collagen aggregation and TXB2 versus placebo $(p=0.05$ and $\mathrm{p}=0.03$, respectively). The authors concluded, 'Highly purified DHA may be a more effective anti-thrombotic agent than EPA.' Thus, supplementing patients with type 2 diabetes with $4 \mathrm{~g}$ of DHA per day may be particularly effective for quickly reducing platelet aggregation, reversing impaired fibrinolysis and improving endothelial dysfunction. ${ }^{25}$ Even so, the REDUCE-IT study found a significant reduction in cardiovascular events in high-risk patients using $4 \mathrm{~g}$ of EPA per day. ${ }^{26}$

The endothelial production of nitric oxide, prostacyclin and tissue-plasminogen activator is very important for preventing platelet aggregation and acute cardiovascular events. ${ }^{27}$ By damaging the endothelium, consuming isolated sources of LA may actually induce a hypercoagulable state, whereas fish oil has been shown to improve endothelial function ${ }^{28}$ and enhance fibrinolytic activity. ${ }^{29}$ DHA, but not EPA, has been found to improve endothelial function, which may be why DHA has been found to have better antihypertensive effects. ${ }^{30}$ Importantly, in healthy patients, a DHA dose of $6 \mathrm{~g}$ /day may be required to significantly reduce platelet aggregation ${ }^{31} 32$ as 1.62 and $1.68 \mathrm{~g}$ of DHA/day have been found ineffective in this regard. 3334

Supplementing the diet with $500 \mathrm{~g}$ (about $17.5 \mathrm{oz}$ ) of oily fish per week for 4 weeks significantly reduces platelet-monocyte aggregates by $35 \%$ versus control, which reverted back to baseline values 4 weeks after discontinuation. ${ }^{35}$ Platelet-monocyte aggregates may promote atherosclerosis and induce inflammatory cytokine, chemokine and adhesion molecule expression. In fact, the authors concluded, 'Our results suggest that reduced platelet activation could represent an important mechanism through which dietary fish confer their putative cardiovascular benefits. ${ }^{35} \mathrm{EPA}$ has also been found to reduce P-selectin, oxidised low-density lipoprotein (LDL) antibodies and glycoprotein IIb/IIIa expression on platelets. ${ }^{36}$ Another report found that $6.6 \mathrm{~g}$ of omega-3 PUFA reduces serum P-selectin expression suggesting a decrease in platelet activation. ${ }^{37}$ The authors noted, 'Most previous studies assessing the effects of fish oils on platelet function have used older techniques with limited reproducibility and physiological relevance. ${ }^{37}$

\section{MARINE OMEGA-3S DO NOT INCREASE THE RISK OF BLEEDS, AND MAY REDUCE THEM}

Regarding safety and bleeding with omega-3s, Dr William Harris summarised the evidence nicely in a 2007 publication. The paper included patients undergoing major 
surgeries ( 2 studies were in coronary artery bypass graft patients, 2 studies in carotid endarterectomy and 15 studies in femoral artery catheterisation) and Dr Harris concluded, 'In these studies, the risk for clinically significant bleeding was virtually nonexistent. ${ }^{38} \mathrm{He}$ also cites a study showing that giving pregnant women $2.7 \mathrm{~g} /$ day of omega-3 does not increase blood loss at delivery. ${ }^{39}$ Dr Harris concluded, 'Thus, the experience has been virtually unanimous: omega-3 fatty acid supplements do not increase the risk for clinically significant bleeding, even in patients also being treated with antiplatelet or antithrombotic medications.' Dr Harris considered the level of evidence supporting this notion as the highest we have in medicine (level of evidence A, from well-designed RCTs). ${ }^{38}$

A recent randomised trial further contradicts the longheld clinical assumption that omega-3 fatty acids increase risk of bleeding during and/or surgery. In the OPERA study, 1516 patients scheduled for cardiac surgery were randomly assigned to matching placebo or fish oil (EPA+DHA; 8-10 g over 2-5 days before surgery, then 2 $\mathrm{g}$ /day on the morning of the surgery until discharge). The omega-3 supplementation did not increase the risk of perioperative bleeding and, unexpectedly, significantly reduced the number of units of blood transfused. ${ }^{40}$ The authors concluded, 'Higher achieved omega-3 PUFA levels were associated with lower risk of bleeding. ${ }^{40}$

\section{A HIGH OMEGA-6/0MEGA-3 RATIO INCREASES PLATELET AGGREGATION}

Compared with saturated fat plus trans fat, a meta-analysis of RCTs found an increased risk of all-cause mortality, coronary heart disease mortality and cardiovascular events with omega-6 industrial seed oils. ${ }^{41}$ This may have to do with the metabolites of omega-6 PUFAs being largely proinflammatory/proaggregatory. ${ }^{42}$ Indeed, omega-3 and omega-6 PUFAs are supposed to balance each other out when they are consumed in the diet at a ratio of around 1 to $1 .^{43}$ However, the increase in our omega-6/ omega-3 ratio has shifted the balance into a proinflammatory/proaggregatory state. Despite LA's ability to lower LDL levels, it can increase LDL susceptibility to oxidation and lipid peroxidation levels ${ }^{445}$ and hence may actually increase the risk of coronary artery disease ${ }^{46}$ as the peroxidation of LA in LDL is thought to be one of the earliest promoters of atherosclerosis. More importantly, oxidised metabolites of LA can increase thrombosis and vasoconstriction by reducing prostacyclin in the vascular wall and increasing TXA2 ${ }^{47}$ Moreover, consuming LA from industrial seed oils may even increase the susceptibility to fatal arrhythmias. $^{48}$

One cross-over study compared a low-erucic acid rapeseed oil (canola oil) versus high-oleic acid sunflower oil to see if there were any differences on platelet aggregation by using oils with a high versus a low LA/ALA ratio. The canola oil provided an omega-6/omega-3 ratio of just 2.8 , whereas those given the high-oleic sunflower oil were provided with an omega-6/omega-3 ratio of 28. In those provided a high omega-6/omega-3 ratio using the higholeic sunflower oil there was an increase in platelet aggregation versus the low omega-6/omega-3 canola oil group. Platelet aggregation was also enhanced in the high-oleic sunflower oil group versus the baseline habitual diet. Thus, even a high-oleic acid omega- 6 industrial seed oil may increase cardiovascular risk. The authors noted that as the omega-6 (LA) to omega-3 (ALA) ratio increased so did platelet aggregation. ${ }^{13}$

In summary, the long-chain omega-3 PUFAs EPA and DHA have antiplatelet effects, but do not increase the risk of clinically significant bleeds and may even reduce the risk of bleeding in the surgical setting, whereas the omega-6 PUFA LA has little effect on reducing platelet aggregation and in some instances may even increase platelet activation. Omega-6 industrial seed oils, as well as suboptimal intakes of marine omega-3s, may increase the risk of thrombotic cardiovascular events. This has been suggested in numerous randomised clinical studies in humans.

\section{Contributors All authors contributed to the final manuscript.}

This research received no specific grant from any funding agency in the public, commercial or not-for-profit sectors.

Competing interests JJDN is the author of The Salt Fix and Superfuel. JOK has an ownership interest in CardioTabs, a company that sells omega-3 supplements.

Provenance and peer review Not commissioned; internally peer reviewed.

Open access This is an open access article distributed in accordance with the Creative Commons Attribution Non Commercial (CC BY-NC 4.0) license, which permits others to distribute, remix, adapt, build upon this work non-commercially, and license their derivative works on different terms, provided the original work is properly cited, appropriate credit is given, any changes made indicated, and the use is non-commercial. See: http://creativecommons.org/licenses/by-nc/4.0/.

\section{REFERENCES}

1. DiNicolantonio JJ. Increase in the intake of refined carbohydrates and sugar may have led to the health decline of the Greenland Eskimos. Open Heart 2016;3:e000444.

2. Kristensen SD, Schmidt EB, Dyerberg J. Dietary supplementation with n-3 polyunsaturated fatty acids and human platelet function: a review with particular emphasis on implications for cardiovascular disease. J Intern Med Suppl 1989;731:141-50.

3. Gao L-gen, Cao J, Mao Q-xia, et al. Influence of omega-3 polyunsaturated fatty acid-supplementation on platelet aggregation in humans: a meta-analysis of randomized controlled trials. Atherosclerosis 2013;226:328-34.

4. Lev El, Solodky A, Harel N, et al. Treatment of aspirin-resistant patients with omega- 3 fatty acids versus aspirin dose escalation. $J$ Am Coll Cardiol 2010;55:114-21.

5. Vanschoonbeek K, Feijge MAH, Paquay M, et al. Variable hypocoagulant effect of fish oil intake in humans: modulation of fibrinogen level and thrombin generation. Arterioscler Thromb Vasc Biol 2004;24:1734-40.

6. Miller ME, Anagnostou AA, Ley B, et al. Effect of fish oil concentrates on hemorheological and hemostatic aspects of diabetes mellitus: a preliminary study. Thromb Res 1987;47:201-14.

7. Cartwright IJ, Pockley AG, Galloway JH, et al. The effects of dietary omega-3 polyunsaturated fatty acids on erythrocyte membrane phospholipids, erythrocyte deformability and blood viscosity in healthy volunteers. Atherosclerosis 1985;55:267-81.

8. Bach R, Schmidt U, Jung F, et al. Effects of fish oil capsules in two dosages on blood pressure, platelet functions, haemorheological and clinical chemistry parameters in apparently healthy subjects. Ann Nutr Metab 1989;33:359-67.

9. Li XL, Steiner M. Fish oil: a potent inhibitor of platelet adhesiveness. Blood 1990;76:938-45. 
10. Andrioli G, Carletto A, Guarini P, et al. Differential effects of dietary supplementation with fish oil or soy lecithin on human platelet adhesion. Thromb Haemost 1999;82:1522-7.

11. Kabir M, Skurnik G, Naour N, et al. Treatment for 2 Mo with N 3 polyunsaturated fatty acids reduces adiposity and some atherogenic factors but does not improve insulin sensitivity in women with type 2 diabetes: a randomized controlled study. Am J Clin Nutr 2007;86:1670-9.

12. DiNicolantonio JJ, Meier P, O'Keefe JH. Omega-3 polyunsaturated fatty acids for the prevention of cardiovascular disease: do formulation, dosage \& comparator matter? Mo Med 2013;110:495-8.

13. Freese R, Mutanen M, Valsta LM, et al. Comparison of the effects of two diets rich in monounsaturated fatty acids differing in their linoleic/alpha-linolenic acid ratio on platelet aggregation. Thromb Haemost 1994;71:73-7.

14. Mutanen M, Freese R, Valsta LM, et al. Rapeseed oil and sunflower oil diets enhance platelet in vitro aggregation and thromboxane production in healthy men when compared with milk fat or habitual diets. Thromb Haemost 1992;67:352-6.

15. Pérez-Jiménez F, Castro $P$, López-Miranda J, et al. Circulating levels of endothelial function are modulated by dietary monounsaturated fat. Atherosclerosis 1999;145:351-8.

16. Sirtori CR, Tremoli E, Gatti E, et al. Controlled evaluation of fat intake in the Mediterranean diet: comparative activities of olive oil and corn oil on plasma lipids and platelets in high-risk patients. Am J Clin Nutr 1986;44:635-42.

17. Navarro MD, Hortelano P, Periago JL, et al. Effect of dietary olive and sunflower oils on the lipid composition of the aorta and platelets and on blood eicosanoids in rats. Arterioscler Thromb 1992;12:830-5.

18. Lahoz C, Alonso R, Ordovás JM, et al. Effects of dietary fat saturation on eicosanoid production, platelet aggregation and blood pressure. Eur J Clin Invest 1997;27:780-7.

19. Rasmussen O, Thomsen C, Ingerslev J, et al. Decrease in von Willebrand factor levels after a high-monounsaturated-fat diet in non-insulin-dependent diabetic subjects. Metabolism 1994;43:1406-9.

20. von Schacky C. Prophylaxis of atherosclerosis with marine omega-3 fatty acids. A comprehensive strategy. Ann Intern Med 1987;107:890-9

21. Tremoli E, Eligini S, Colli S, et al. N-3 fatty acid ethyl ester administration to healthy subjects and to hypertriglyceridemic patients reduces tissue factor activity in adherent monocytes. Arterioscler Thromb 1994;14:1600-8.

22. Marckmann $P$, Jespersen J, Leth $T$, et al. Effect of fish diet versus meat diet on blood lipids, coagulation and fibrinolysis in healthy young men. J Intern Med 1991;229:317-23.

23. DiNicolantonio JJ, Niazi AK, McCarty MF, et al. Omega-3s and cardiovascular health. Ochsner J 2014;14:399-412.

24. Avellone G, Garbo D V, Cordova R, et al. Effects of Mediterranean diet on lipid, coagulative and fibrinolytic fibrinolytic parameters in two randomly selected population samples in Western Sicily. Nutr Metab Cardiovasc Dis 1998:8:287-96.

25. Woodman RJ, Mori TA, Burke V, et al. Effects of purified eicosapentaenoic acid and docosahexaenoic acid on platelet, fibrinolytic and vascular function in hypertensive type 2 diabetic patients. Atherosclerosis 2003;166:85-93.

26. Bhatt $\mathrm{DL}$, Steg $\mathrm{PG}$, Miller $\mathrm{M}$, et al. Cardiovascular risk reduction with Icosapent ethyl for hypertriglyceridemia. N Engl J Med 2019;380:11-22.

27. Sagripanti A, Carpi A. Antithrombotic and prothrombotic activities of the vascular endothelium. Biomed Pharmacother 2000;54:107-11.

28. Chin JP, Dart AM. How do fish oils affect vascular function? Clin Exp Pharmacol Physiol 1995;22:71-81.
29. Knapp HR. Dietary fatty acids in human thrombosis and hemostasis. Am J Clin Nutr 1997;65(5 Suppl):1687S-98.

30. Mori TA, Watts GF, Burke V, et al. Differential effects of eicosapentaenoic acid and docosahexaenoic acid on vascular reactivity of the forearm microcirculation in hyperlipidemic, overweight men. Circulation 2000;102:1264-9.

31. von Schacky C, Weber PC. Metabolism and effects on platelet function of the purified eicosapentaenoic and docosahexaenoic acids in humans. J Clin Invest 1985;76:2446-50.

32. Ferretti A, Nelson GJ, Schmidt PC, et al. Dietary docosahexaenoic acid reduces the thromboxane/prostacyclin synthetic ratio in humans. The Journal of Nutritional Biochemistry 1998;9:88-92.

33. Agren JJ, Väisänen $\mathrm{S}$, Hänninen $\mathrm{O}$, et al. Hemostatic factors and platelet aggregation after a fish-enriched diet or fish oil or docosahexaenoic acid supplementation. Prostaglandins Leukot Essent Fatty Acids 1997;57:419-21

34. Conquer JA, Holub BJ. Supplementation with an algae source of docosahexaenoic acid increases (n-3) fatty acid status and alters selected risk factors for heart disease in vegetarian subjects. $J$ Nutr 1996;126:3032-9.

35. Din JN, Harding SA, Valerio CJ, et al. Dietary intervention with oil rich fish reduces platelet-monocyte aggregation in man. Atherosclerosis 2008;197:290-6.

36. Nomura S, Kanazawa S, Fukuhara S. Effects of eicosapentaenoic acid on platelet activation markers and cell adhesion molecules in hyperlipidemic patients with type 2 diabetes mellitus. J Diabetes Complications 2003;17:153-9.

37. Eschen O, Christensen JH, De Caterina R, et al. Soluble adhesion molecules in healthy subjects: a dose-response study using n-3 fatty acids. Nutr Metab Cardiovasc Dis 2004;14:180-5.

38. Harris WS. Expert opinion: Omega-3 fatty acids and bleeding-cause for concern? Am J Cardiol 2007;99:S44-S46.

39. Olsen SF, Sørensen JD, Secher NJ, et al. Randomised controlled tria of effect of fish-oil supplementation on pregnancy duration. Lancet 1992;339:1003-7.

40. Akintoye $E$, Sethi $P$, Harris WS, et al. Inisights from the opera randomized trial. Cardiovascular Quality and Outcomes 2018;11.

41. Ramsden CE, Zamora D, Leelarthaepin B, et al. Use of dietary linoleic acid for secondary prevention of coronary heart disease and death: evaluation of recovered data from the Sydney diet heart study and updated meta-analysis. BMJ 2013;346:e8707.

42. DiNicolantonio $\mathrm{JJ}, \mathrm{O}^{\prime}$ Keefe $\mathrm{JH}$. Importance of maintaining a low omega-6/omega-3 ratio for reducing inflammation. Open Heart 2018;5:e000946.

43. Simopoulos AP, DiNicolantonio JJ. The importance of a balanced $\omega-6$ to $\omega-3$ ratio in the prevention and management of obesity. Open Heart 2016;3:e000385.

44. Jenkinson A, Franklin MF, Wahle K, et al. Dietary intakes of polyunsaturated fatty acids and indices of oxidative stress in human volunteers. Eur J Clin Nutr 1999;53:523-8.

45. Berry EM, Eisenberg S, Haratz D, et al. Effects of diets rich in monounsaturated fatty acids on plasma lipoproteins--the Jerusalem Nutrition Study: high MUFAs vs high PUFAs. Am J Clin Nutr 1991;53:899-907.

46. Hodgson JM, Wahlqvist ML, Boxall JA, et al. Can linoleic acid contribute to coronary artery disease? Am J Clin Nutr 1993;58:228-34.

47. Umeda F, Kunisaki M, Inoguchi T, et al. Modification of prostacyclinstimulatory activity in sera by glucose, insulin, low density lipoprotein, linoleic acid and linoleic acid hydroperoxide. Diabetes Res Clin Pract 1990;8:137-44.

48. Li Y, Kang JX, Leaf A. Differential effects of various eicosanoids on the production or prevention of arrhythmias in cultured neonatal rat cardiac myocytes. Prostaglandins 1997;54:511-30. 